\title{
Case Report \\ Unusual presentation of a massive pseudo pancreatic cyst
}

Raviraj Subramaniam, Krishnakumar Arooran

Teaching Hospital, Jaffna, Sri Lanka

Keywords: Pancreas, Pancreatic pseudocyst, Cyst gastrostomy

Corresponding Author: K W D A Anuradha, E-mail: < dr.S.raviraj@gmail.com >
Received: January 2018, Accepted revised version June 2018, Published: June 2018
Competing Interests: Authors have declared that no competing interests exist
C) Authors. This is an open-access article distributed under a Creative Commons Attribution-Share Alike
4.0 International License (CC BY-SA 4.0), which permits unrestricted use, distribution, and reproduction in any
medium, provided the original author and source are attributed and materials are shared under the same
license.

\section{Introduction}

Pseudocyst of the pancreas is the most common complication of acute or chronic pancreatitis [1]. It is a common clinical entity with an incidence of $30-40 \%$ in chronic pancreatitis [2]. A pseudocyst is a localized fluid collection rich in pancreatic enzymes including amylase, surrounded by a fibrous tissue wall but not lined by epithelium. Pseudocysts commonly connect with the pancreatic duct system [1,2] but occasionally not. Symptomatic pseudocysts and complicated cysts need intervention. The timing and choice of surgical intervention depend on various factors. The D'Egidio classification of pancreatic pseudocyst is widely practiced in clinical settings [1].

We present a case of a 47-year-old male patient with a massive pancreatic pseudocyst who underwent cyst gastrostomy.

\section{Case presentation}

A 47-year old man was admitted with progressive gross abdominal distension of six months duration and vomiting after meals of two months duration. On direct questioning, he admitted to upper abdominal pain radiating to the back, on and off for one-year, interfering with his sleep. Pain was relieved by bending forward and was not related to fatty meals. Vomitus contained food particles but was not bile stained. He had no history of haematemesis. He gave a history of consuming half a bottle of alcohol daily for 20 years. He had no history of yellowish discolouration of sclera, passing tea coloured urine, pale stools or itching. He had no steatorrhoea. There was no history of polyuria or polydipsia. He had no previous admission to hospital regarding this problem. He was treated by a general practitioner for chronic liver cell disease (CLCD) as he complained of abdominal distension and was suspected to have CLCD with ascites.

General examination revealed anaemia without icterus or cachexia. Abdominal examination revealed a grossly distended abdomen but no clinical evidence of CLCD. Percussion showed 
dullness over most of the abdomen. There were no other significant positive findings in the systemic examination.

The full blood count showed a neutrophil leukocytosis. Liver profile and renal profile were normal. Cardiac and respiratory functions were normal. Ultrasonography (US) and contrast enhanced computerised axial tomography of the abdomen revealed acute on chronic pancreatitis with large pseudocyst formation along the body and tail of the pancreas, measuring $16 \mathrm{~cm} \times 12 \mathrm{~cm}$ and another smaller pseudocyst in the head of the pancreas (Figure 1).

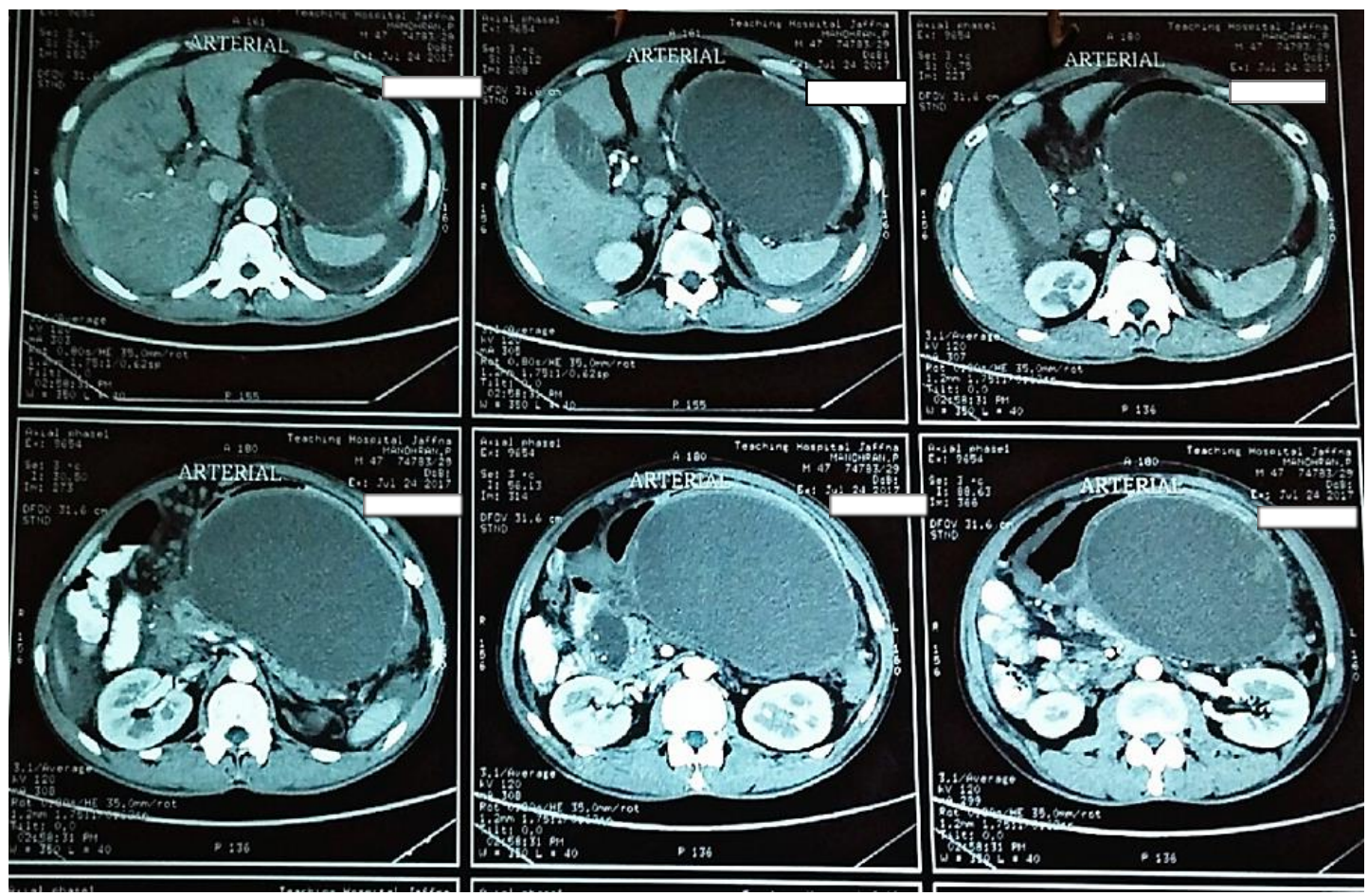

Figure 1: Contrast enhanced computerised axial tomography of abdomen showing pancreatic pseudocyst.

A diagnosis of pseudocyst of the pancreas was made and the patient was informed regarding the disease. After obtaining consent, cyst gastrostomy was done to prevent further complications. The post-operative period was uneventful. The patient was referred to a consultant psychiatrist for alcohol withdrawal symptoms and to a pain clinic to alleviate the pain of chronic pancreatitis.

\section{Discussion}

Patients with pancreatic pseudocysts commonly give a history of repeated admissions to hospital due to severe abdominal pain, but this patient had no history of such admissions. Excessive alcohol intake and the presence of gross abdominal distension resulted in him being managed as a case of CLCD. 
The prevalence of pancreatic pseudocysts in acute pancreatitis has been reported to range from $6 \%$ to $18.5 \%$ [4]. The prevalence of pseudocysts in chronic pancreatitis is $20 \%$ to $40 \%$ [4]. Pancreatic pseudocysts are most commonly seen in alcoholic chronic pancreatitis (70\% to 78\%) [4]. The second highest incidence is in idiopathic chronic pancreatitis (6\% to $16 \%$ ) followed by biliary pancreatitis (6\% to $80 \%$ ) [4].

The diagnosis of pancreatic pseudocyst is established mainly by imaging studies. Among these, abdominal ultrasonography is an important initial investigation [4] as seen in this patient. The sensitivity of ultrasound in the detection of pancreatic pseudocysts is 75 to $90 \%$ [2]. Computerized tomography (CT) is the imaging method of choice with $82 \%$ to $100 \%$ sensitivity and $98 \%$ specificity $(2,4)$. In this patient, the diagnosis of pseudo cyst was finally confirmed by CT. Endoscopic ultrasonography (EUS) is useful to distinguish acute fluid collections from pancreatic abscess and acute pancreatic pseudocyst. EUS has the highest sensitivity (93\% to 100\%) and specificity (92\% to 98\%) [4]. Diagnostic puncture and cytology under EUS guidance helps to differentiate cystic malignances from pseudocyst (4). Fluid aspirated from the pseudocyst can be tested for enzymes (amylase and lipase) and tumour markers (CEA, CA 199-9, CA 125 and CA 72-4) [4]. Magnetic resonance imaging (MRI) and magnetic resonance cholangiopancreatography (MRCP) are highly sensitive diagnostic tools for pancreatic pseudo cyst but most centres do not routinely use these techniques because CT scans offer most of the required information [4]. MRI is highly sensitive to detect bleeding or complex fluid collections in pancreatic pseudocyst [4].

Pancreatic pseudocysts are classified in several ways. The most widely referenced classification is the one by D'Egidio et al. This classification identifies three distinct types of pseudocysts [4].

Type 1- Acute post necrotic pseudocyst which occurs after an episode of acute pancreatitis and is associated with normal duct anatomy and rarely communicates with the pancreatic duct.

Type 2- Also known as "post necrotic pseudo cyst". It occurs after an episode of acute or chronic pancreatitis and there is often duct pseudocyst communication.

Type 3- Defined as "retention" pseudocysts which occur in chronic pancreatitis. It is usually associated with duct stricture and pseudocyst duct communication.

The pseudocyst in this patient belong to Type 2 in this classification.

Complications of pancreatic pseudocysts include gastric outlet obstruction or duodenal stenosis, stenosis of the common bile duct, splenic vein thrombosis, splenic infarct and bleeding, portal hypertension, rupture of pseudocyst, compression of major abdominal vessels, infection of the pseudocyst, haemorrhage into the pseudocyst and pancreatic pleural fistula. This patient had only gastric outlet obstruction due to compression of the pylorus of the stomach $[2,3,4]$. 
Indications for surgical intervention include all the complications mentioned above. Asymptomatic pancreatic pseudocyst of more than $6 \mathrm{~cm}$ without any regression after more than 6 weeks is also considered in most centres as an indication for interference [4]. The options for drainage are external percutaneous drainage guided by US or CT, EUS-guided aspiration, endoscopic drainage (transpapillary approach with ERCP or direct drainage across the stomach or duodenum) or laparotomy and drainage of the cyst into the stomach, duodenum or jejunum. Percutaneous drainage results in higher mortality and morbidity rates and longer hospital stays than surgical treatment of pancreatic pseudocyst, especially in non-elective patient [5]. This patient underwent open cyst gastrostomy.

\section{Conclusion}

The unusual presentation in this patient delayed the correct management and increased the risk of morbidity and mortality. Pancreatic pseudocyst have multiple treatment modalities which have to be selected on an individual basis based on the clinical and radiological findings and the available facilities.

\section{References}

1. Zhang $A B$, Zheng SS. Treatment of pancreatic pseudocysts in line with $D^{\prime}$ Egidio's classification. World Journal of Gastroenterology: WJG. 2005 Feb 7;11(5):729.

2. Habashi S, Draganov PV. Pancreatic pseudocyst, World J Gastroenterol. 2009 Jan 7; 15(1): 38-47.https://doi.org/10.3748/wjg.15.38

3. Accetta, P. et al. (2010) 'Pseudocisto pancreático com envolvimento esplênico: relato de caso', Revista do Colégio Brasileiro de Cirurgiões. Colégio Brasileiro de Cirurgiões, 37(6), pp. 457-459. https://doi.org/10.1590/S0100-69912010000600014

4. Lerch MM, Stier A, Wahnschaffe U, Mayerle J. Pancreatic pseudocysts: observation, endoscopic drainage, or resection? Deutsches Ärzteblatt International. 2009 Sep;106(38):614. https://doi.org/10.3238/arztebl.2009.0614

5. Heider R, Meyer AA, Galanko JA, Behrns KE. Percutaneous drainage of pancreatic pseudocysts is associated with a higher failure rate than surgical treatment in unselected patients. Annals of surgery. 1999 Jun;229(6):781. (Accessed: 8 October 2017). 